\title{
Neural Network based Fault Diagnosis in Analog Electronic Circuit using Polynomial Curve Fitting
}

\author{
Ashwani Kumar \\ Electronics and Communication Engineering \\ Section \\ Yadavindra College of Engineering, Punjabi \\ University, Guru Kashi Campus, Talwandi Sabo
}

\author{
A.P.Singh \\ Department of Electronics and Communication \\ Engineering \\ Sant Longowal Institute of Engineering and \\ Technology, Longowal
}

\begin{abstract}
Many studies have been presented for the fault diagnosis of electronic analog circuits with worst case fault models using $\pm 50 \%$ variation in the parametric values of the components. The study of for parametric fault detection in electronic analog circuits -faults as small as $10 \%$ or less was uncovered. The use of the neural network for parametric fault diagnosis in an analog circuit, based upon the polynomial curve fitting coefficients of the output response of an analog circuit is presented in this study. Building upon the theory of polynomial coefficients we propose a parametric fault diagnosis methodology. A polynomial of suitable degree is fitted to the output frequency response of an analog circuit. The coefficients of the polynomial attain different values under faulty and non faulty conditions. Using these features of polynomial coefficients, a BPNN is used to detect the parametric faults. Simulation results are presented for a benchmark bi quad filter circuit. Single resistance and capacitance faults of $\pm 1 \%$ to $\pm 50 \%$ deviation from nominal values were correctly diagnosed.
\end{abstract}

\section{Keywords}

Neural Network, Parametric faults, Analog circuit, Curve fitting, Polynomial coefficients.

\section{INTRODUCTION}

Whenever a system does not behave normally as it should, means system is faulty. Fault diagnosis involves two steps. One the detection of fault in the system is called fault detection. Second to locate the position of the fault is called fault isolation. Electronic circuits and systems have become part and parcel of our daily life. Major business issues and customer expectations for electronic systems are zero system failure, high reliability and longevity. Fault diagnosis in analog electronic circuits is a challenging research area. Tolerance effects of analog components, lack of test nodes, presence of feedback loops, nonlinearity problems etc. make the diagnosis in analog circuits difficult.

Several methods have been presented for parametric fault diagnosis of analog circuits and systems [1]. A survey of the research conducted in this area clearly indicates that analog fault diagnosis is complicated due to the poor fault models, component tolerances, and nonlinearity issues. The artificial neural network is an efficient approach to fault diagnosis due to its robustness and strong learning ability.

Fault diagnosis methods are classified as simulation before test (SBT) and simulation after test (SAT). Simulation before test approach is more effective as it eliminates on line simulation process and needs only once off- line computational effort before test activity. Fault dictionary is a practical approach belonging to the simulation before test techniques. An input stimulus is first selected to excite the CUT. The circuit response to the input excitation is then simulated/measured at different faulty and non faulty conditions.

Fault dictionary approach based on SBT is generally used. Different feature extraction techniques to build the fault dictionary for the fault diagnosis of analog electronic circuits under SBT method has been given by different researchers. Since limited output nodes are available to have the output response, so for the fault diagnosis needs unique features which represent the faulty and non faulty nature of the analog electronic circuit. Along with the unique feature extraction it is also desirable that computational overhead should also be minimum. In [2] output response of the circuit is extracted and principal component analysis is performed to reduce the feature space. The soft and the hard faults of linear analog circuit using slope fault feature and back propagation neural network is given. The soft fault diagnosis based on soft fault feature and back propagation neural network. A linear analog circuit containing only the resistive components has been used as an example circuit [3].

Fault diagnosis of analog circuit using frequency response and neural network as fault classifier are successfully given for single and multiple faults. The peak frequency and peak gain of the frequency response of the analog circuit are used to extract the distinct signatures for faulty and non faulty conditions [4]. The parametric variation in the component has been taken as $\pm 50 \%$ from its nominal values. Ensembles of the neural network for fault diagnosis in analog electronic circuit are described in [5]. Some studies [6,7, 8, and 9] have used the transient response of the analog electronic circuit and wavelet transform as a pre processor for fault signature extraction and finally neural network for fault classification. Some studies used node voltages, DC supply current, and AC response of the CUT, simulation of the circuit in frequency domain and FFT for feature extraction of faulty and non faulty analog circuit [10-16].

In the present fault diagnosis method, fault dictionary approach based on SBT technique has been presented. A benchmark bi quad filter circuit has been used for fault diagnosis. Only the parametric faults of the components are considered in this study. The frequency responses of the circuit under test (CUT) have been plotted using bode plot of MULTISIM software. These frequency plots are fitted with a polynomial of suitable order. Polynomial coefficients of the curve fitted to the frequency response of the CUT are used as features representing the fault and fault free analog circuit. These polynomial coefficients are used to train the neural 
network used for the classification of the faults. All parametric faults of the components with $\pm 1 \%$ to $\pm 50 \%$ deviation (using $\pm 1 \%$ deviation resolution) from nominal values were correctly diagnosed.

The material in this paper is arranged in the following order. In section 2, we briefly discuss proposed methodology for fault diagnosis used in this paper. Section 3, describes experimental validation of fault diagnosis for a bench mark analog circuit. Section 4 and 5 covers result and discussion, conclusion respectively.

\section{PROPOSED METHODOLOGY}

Fault diagnosis in analog electronic circuits is a difficult as most of the analog circuit response is frequency dependent and only output terminal is accessible for most applications in practice. So it is needed that the features extracted from circuit response should be unique under faulty and non faulty conditions. That will result in good fault diagnosis of the analog circuit. The proposed methodology for fault diagnosis integrates in the following basic modular steps as shown in figure1.

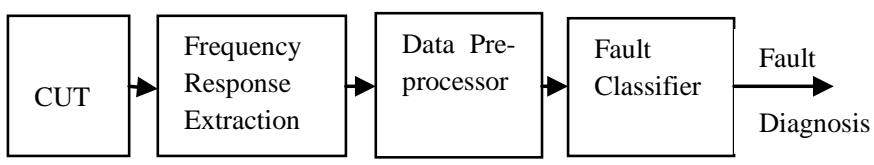

Fig 1: A block diagram for the proposed fault diagnosis methodology.

\subsection{Frequency Response Extraction}

In the proposed methodology the unique feature extraction of the analog circuit is obtained by extracting its frequency response. Frequency response of an analog circuit is the graph which shows the variation in the gain of the analog circuit with respect to the frequency of operation, when the circuit is simulated by a standard stimulus. This proposed methodology is for an analog circuit whose output is frequency dependent. When the frequency of the input stimulus is varied the output voltage and hence the gain of the circuit varies. By plotting this variation in the gain with respect to frequency, a frequency response of the circuit is obtained.

Mathematically to implement this, the transfer function of the analog circuit is required to be calculated depending upon its required response and the band width requirement. The transfer function of the circuit is the ratio of its output voltage to the input voltage i.e. $v_{o} / v_{i}$. The component values are also selected accordingly. Once the transfer function of the analog circuit is calculated, one can easily know the value of the output voltage of the circuit for a known value of the input stimulus. These output voltage obtained for different input frequencies results the overall frequency response of the circuit. The transfer function of the bi quad filter circuit is $H(s)=\frac{G_{p} \omega_{0}^{2}}{s^{2}+\frac{\omega_{0}}{Q} s+\omega_{0}^{2}}$. Where $\mathrm{G}_{\mathrm{p}}, \mathrm{Q}$ is the $\mathrm{Q}$ factor, $\omega_{0}$ is the center frequency and $s=\sigma+j \omega$ is the complex frequency. Band width of the filter circuit is approximated by $B=\omega_{0} / Q$.

Apart from the mathematically obtaining the frequency response of the analog circuit, this can also be obtained by simulating the analog circuit and using the bode plotter. Bode plotter is very useful for the analysis of filter circuits. It produces a graph of circuit's frequency response. In this the gain of the circuit under test is plotted with respect to the frequency. Bode plotter is available as bode diagram of frequency response in the control system tool box of
MATLAB software. This is used to compute the magnitude and phase of the frequency response of the linear time invariant models. In the bode diagram the magnitude is plotted in $\mathrm{db}$ and the phase in degrees. Bode plotter is also available as bode plotter instrument in the MULTISIM software. In this the gain of the circuit under test is plotted with respect to the frequency. An AC source stimulus is required to simulate the circuit. Frequency of the AC source does not affect the function of the bode plotter. But the presence of the AC source is must in the circuit.

The frequency response of the circuit under test is obtained using the bode plotter for faulty and non faulty conditions of the components of the CUT. The parametric fault introduced in each component values are with the variation of $\pm 1 \%$ and up to $\pm 50 \%$ i.e. hundred faults are there for each component keeping all other components at their nominal values within the tolerance limit. It has been observed that for each fault in the circuit component value a unique frequency response graph is obtained. The uniqueness in each of the frequency responses graphs leads further to the fault diagnosis process.

\subsection{Data Preprocessing}

After the circuit simulation, we have different frequency responses graphs indicating the different parametric variation faults in each of the components present in the circuit. The collected graphs are applied to the preprocessor to get the proper and distinguishable features. This is done using polynomial curve fitting. Polynomials are one of the most commonly used types of curves in regression. The Polynomial Curve Fitting uses the method of least squares when fitting data. The fitting process requires a model that relates the response data to the predictor data with one or more coefficients. The result of the fitting process is an estimate of the coefficients of the model. To obtain the coefficient estimates, the least squares method minimizes the summed square of residuals.

The least-squares $j^{\text {th }}$ degree Polynomials method uses $j^{\text {th }}$ degree polynomials $y=a_{0}+a_{1} x+a_{2} x^{2}+\cdots+a_{j} x^{j}$ to approximate the given set of data, $\left(x_{1}, y_{1}\right),\left(x_{2}, y_{2}\right), \ldots\left(x_{k}, y_{k}\right)$, where $k \geq j+1$. The curve of best fit is that for which, $\mathrm{E}$, the sum of squares of the errors is minimum. This is known as the principle of least squares and was suggested by Legendre. For the best fitting curve $f(x)$ has the least square error. i.e.

$$
\begin{aligned}
E & =\sum_{i=1}^{k}\left[y_{i}-f\left(x_{i}\right)\right]^{2} \\
& =\sum_{i=1}^{k}\left[y_{i}-\left(a_{0}+a_{1} x_{i}+a_{2} x_{i}^{2}+\cdots a_{j} x_{i}^{j}\right)\right]^{2}=\min .
\end{aligned}
$$

These unknown coefficients will yield their first derivative zero for Where in equation (i) $x_{i}$ and $y_{i}$ are known values. $a_{0}, a_{1}, a_{2}, \ldots, a_{m}$ are unknown coefficients least square error. 


$$
\left\{\begin{array}{c}
\frac{\partial E}{\partial a_{0}}=2 \sum_{i=1}^{k}\left[y_{i}-\left(a_{0}+a_{1} x_{i}+a_{2} x_{i}^{2}+\cdots a_{j} x_{i}^{j}\right)\right]=0 \\
\frac{\partial E}{\partial a_{1}}=2 \sum_{i=1}^{k} x_{i}\left[y_{i}-\left(a_{0}+a_{1} x_{i}+a_{2} x_{i}^{2}+\cdots a_{j} x_{i}^{j}\right)\right]=0 \\
\frac{\partial E}{\partial a_{2}}=2 \sum_{i=1}^{k} x_{i}^{2}\left[y_{i}-\left(a_{0}+a_{1} x_{i}+a_{2} x_{i}^{2}+\cdots a_{j} x_{i}^{j}\right)\right]=0 \\
\cdot \\
\cdot \\
\frac{\partial E}{\partial a_{j}}=2 \sum_{i=1}^{k} x_{i}^{j}\left[y_{i}-\left(a_{0}+a_{1} x_{i}+a_{2} x_{i}^{2}+\cdots a_{j} x_{i}^{j}\right)\right]=0
\end{array}\right.
$$

After the expansion of the above equations, in equation (ii) we have

$$
\left\{\begin{array}{c}
\sum_{i=1}^{k} y_{i}=a_{0} \sum_{i=1}^{k} 1+a_{1} \sum_{i=1}^{k} x_{i}+a_{2} \sum_{i=1}^{k} x_{i}^{2}+\cdots+a_{j} \sum_{i=1}^{k} x_{i}^{j} \\
\sum_{i=1}^{k} x_{i} y_{i}=a_{0} \sum_{i=1}^{k} x_{i}+a_{1} \sum_{i=1}^{k} x_{i}^{2}+a_{2} \sum_{i=1}^{k} x_{i}^{3}+\cdots+a_{j} \sum_{i=1}^{k} x_{i}^{j+1} \\
\sum_{i=1}^{k} x_{i}^{2} y_{i}=a_{0} \sum_{i=1}^{k} x_{i}^{2}+a_{1} \sum_{i=1}^{k} x_{i}^{3}+a_{2} \sum_{i=1}^{k} x_{i}^{4}+\cdots+a_{j} \sum_{i=1}^{k} x_{i}^{j+2} \\
\vdots \\
\sum_{i=1}^{k} x_{i}^{j} y_{i}=a_{0} \sum_{i=1}^{k} x_{i}^{j}+a_{1} \sum_{i=1}^{k} x_{i}^{j+1}+a_{2} \sum_{i=1}^{k} x_{i}^{j+2}+. .+a_{j} \sum_{i=1}^{k} x_{i}^{2 j}
\end{array}\right.
$$

The polynomial coefficients $a_{0}, a_{1}, a_{2}, \ldots, a_{j}$ can be obtained by solving these above given equation (iii), linear equations.

Polynomial curve fitting is done using the curve fitting toolbox of MATLAB software. Which also based on the theory of least square methods. Since the frequency response graphs are different for different fault senarios, polynomial coefficients are also different and unique for different faults. These polynomial coefficients are used to prepare the fault dictionary, for further classificatin of the faults.

\subsection{Fault Classification}

Fault classification is integrated in two basic steps. Preparation of the fault dictionary for different identified faults in the first step. Fault diagnosis using a proper artificial neural network in the second step. The size of the fault dictionary will be based on number of the identified faults in a component and the order of the polynomial used for curve fitting. If the number of identified faults in a component are $n$ and the order of the polynomial is $m$, then there will be $(n \times$ number of components +1$)$ entries ( $n$ for $n$ number of faults and one for fault free condition) in the fault dictionary and each entry will have $m+1$ number of coefficient for that component.

Artificial Neural Networks, is a parallel distributed processing system made up of highly interconnected neural computing elements. These networks have the ability to learn and there by acquire knowledge. This acquired knowledge makes neural network to solve problems. Neural Networks architectures have been classified into various types based on their learning mechanism. These neurons are connected to form a network and are organised in the form of layers. These are connected by highly synaptic weights.The Artificial Neural Networks (ANN ) have a learning ability as synaptic weights can be strengthened or weakened during the learning process, and in this way, information can be stored in the neural network. Each neuron has activation function, as a function of inputs it has received. A neuron sends its activation as a signal to several other neurons. It is important to note that a neuron can send only one signal at a time, although signals are broadcasted to several other neurons.

The artificial neural network used in proposed methodology is a multilayer backpropagation neural network. This type of neural network is used in patteren recognition, classification, function approximation etc. The artificial neural network model with multiple inputs and one output for the fault diagnosis in analog circuit is shown in figure2. Here $\mathrm{X}=\left[\mathrm{X}_{1}, \mathrm{X}_{2}, \mathrm{X}_{3}, \ldots \ldots \mathrm{X}_{\mathrm{n}}\right]$ is the input vector and $\mathrm{n}$ is the number of neurons in the input layer. $Y_{k}$ is the output of the neural network. Where $\mathrm{k}$ represent the different coponents present in the analog circuit.

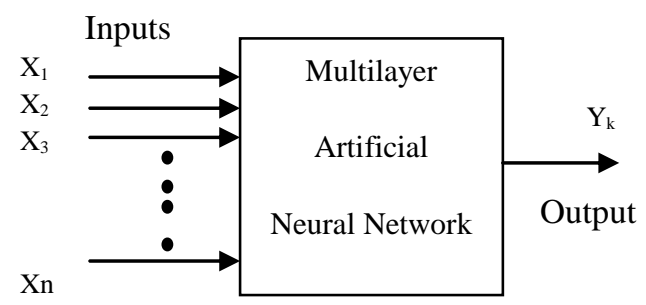

Fig 2: Proposed model of artificial neural network for the fault diagnosis in analog circuits

$X_{1} \ldots \ldots . X_{n}$ represents the coefficients of the polynomial fitted to the output frequency response of the analog circuit. $Y_{k}$ is the output of the neural network which represent the variation in the component vaule from $\pm 1 \%$ to $\pm 50 \%$. For every component there are 101 samples of input polynomial coefficients and corresponding 101 outputs. So the total number of input-output samples for ANN will be $(100 \times$ number of components +1$)$. These includes 100 fault samples for each component and one sample for the nominal value. In this work a neural network with three layer strctural architecture has been proposed. Input layer, output layer and hidden layer. The nodes in the input layer is equal to the number of coefficients, and the number of nodes in the middle hidden layers are varied to achieve the best diagnosis classification. Neural network weights are adapted during the training. Evaluation of the fault diagnosis classification is evaluated during the test phase.

\section{EXPERIMENTAL VALIDATION}

The proposed methodology described in the previous section is applied to the benchmark bi quad filter circuit as the circuit under test. As this circuit has already been used previously in other fault diagnosis studies [17], [18] and has been found to fairly tolerant to component variations, we found it to be a suitable choice.

\subsection{Circuit Simulation}

We have taken up the benchmark bi quad filter circuit shown in figure 3 to illustrate our approach to fault diagnosis. The component values are $\mathrm{C} 1=\mathrm{C} 2=10 \mathrm{nf}, \mathrm{R} 1=\mathrm{R} 3=2.7 \mathrm{~K} \Omega$, $\mathrm{R} 2=1.5 \mathrm{~K} \Omega, \quad \mathrm{R} 4=12 \mathrm{~K} \Omega, \quad \mathrm{R} 5=1 \mathrm{~K} \Omega \quad$ and $\mathrm{R} 6=10 \mathrm{~K} \Omega$. The resistors and capacitors are assumed to have the tolerance 
values $5 \%$ and $10 \%$ respectively. In order to verify the correctness of our proposed methodology, the software MULTISIM and MATLAB are adopted to implement the fault diagnosis process. The analog circuit is simulated using MULTISIM software and the frequency response of the circuit under test is obtained by its bode plotter instrument.

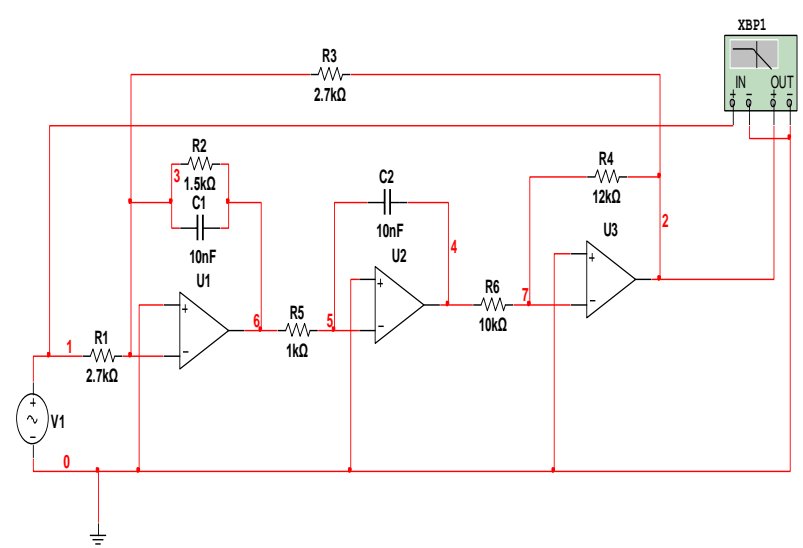

Fig 3: Bi quad filter circuit (Crcuit Under Test)

Hundred soft faults ( for the increase and decrease in the parametric value) are simulated for each components $(\mathrm{C} 1, \mathrm{C} 2, \mathrm{R} 1, \mathrm{R} 2, \mathrm{R} 3, \mathrm{R} 4)$. The component parametric values are tolerated by $+1 \%$ (increase) and $-1 \%$ (decrease) faults respectively. The maximum variation in the parametric value is limited to $\pm 50 \%$. Total 101 frequency responses, hundred for the fault classes and one for the nominal value of the component, are recorded for the components of the circuit under test. It has been observed that each response shows a change with the change in the parametric value of the components. This distinction in frequency response feature of CUT for the component value serves the basis of fault diagnosis process. These frequency responses are stored in the form of excel files.

\subsection{Data Preprocessing}

The feature extraction for the purpose of the fault diagnosis is accomplished with the data preproceesing. The simulation results are preprocessed using the proposed methodology described in section 2.2.for the preparation of the fault dictionary. For all the faults for the components of the circuit under test, polynomial curve fitting method is applied to get the basic polynomial coefficients. The curve fitting is impemented by using the curve fitting tool box of the MATLAB software. The plonomial curve fitting tool box of MATLAB uses the least square method. The following steps are following in the curve fitting process.

1. The frequency response graph is first transferred and stored in an microsoft office excel work sheet.

2. These values of the graph from excel worksheet are tranferred to the matlab workspace.

3. The data stored in the matlab workspace is imported in the curve fitting tool box.

4. The imported graph in the curve fiiting tool box is curve fitted using polynomial curve fitting.

5. A ninth order polynomial is used to fit output frequency response of CUT yielding nine polynomial coefficients.
6. This process is repeated for every output frequency response related to each fault senario of each component of CUT and nine polynomial coefficients are recorded for each curve fit. These coefficients are further used to build the fault dictionary. The figure 4 shows an example curve fit for a fault senario in component $\mathrm{C} 1$.

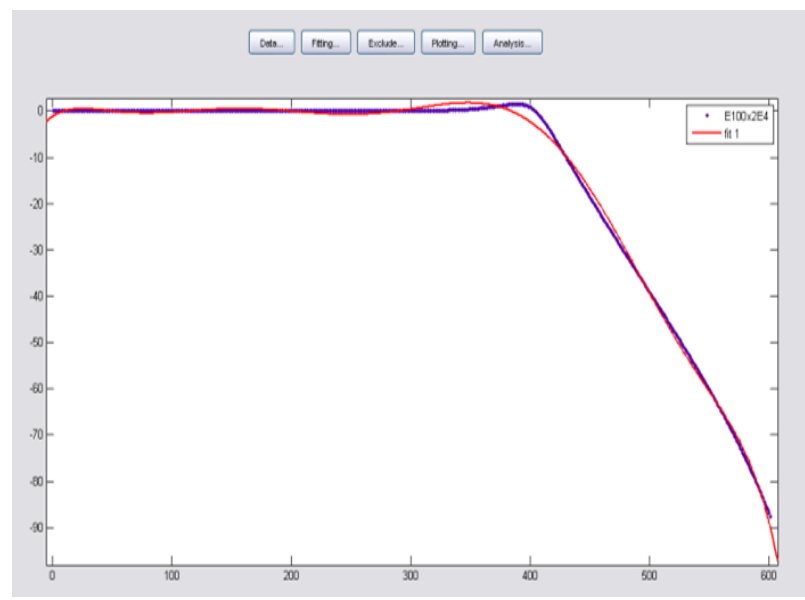

Fig 4: Curve fit example of fault senario in component $\mathrm{C} 1$

\subsection{Fault Classification}

The first step in fault classification procedure is the identification of the faults and preparing the fault dictionary. The output response of the circuit is curve fitted with $9^{\text {th }}$ order polynomial.For fault classification purpose we use ten polynomial coefficients. The fault classification results are fairly good by using these coeffiecients. Hence for every component in the circuit a $10 \times 101$ fault dictionary matrix is prepared. For simplicity in the fault dictionary plots of the coefficients naming P1 and P2 are shown in figure 5. These plots show the polynomial coefficient values with respect to the change in the $\%$ variation in the component value.

The second step is the design of proper design of neural network for the diagnosis of the faults. The NN toolbox of MATLAB software is used to design the neural network architecture for fault classification. In the experimental work the neural network degined contains three layered structure, input layer, output layer and one hidden layer as shown in figure 6 .

There are $h$ numbers of hidden layer neurons and $B_{1}=\left[b_{1}, b_{2}, b_{3}, \ldots \ldots . ., b_{h}\right]$ is the bias vector of the hidden layer neurons. Output layer contains one output neuron with their bias vector $\mathrm{b}_{\mathrm{o}}$. The output $O_{h}$ of the $h^{\text {th }}$ neuron in the hidden layer is given by

$o_{h}=f 1\left(\sum_{k=1}^{2} W_{i n} X_{k}+b_{h}\right)$

The output $Y_{k}$ of the output neuron in the output layer is

$Y_{k}=f 2\left(\sum_{i=1}^{h} W_{j i} O_{h}+b_{o}\right)$ 


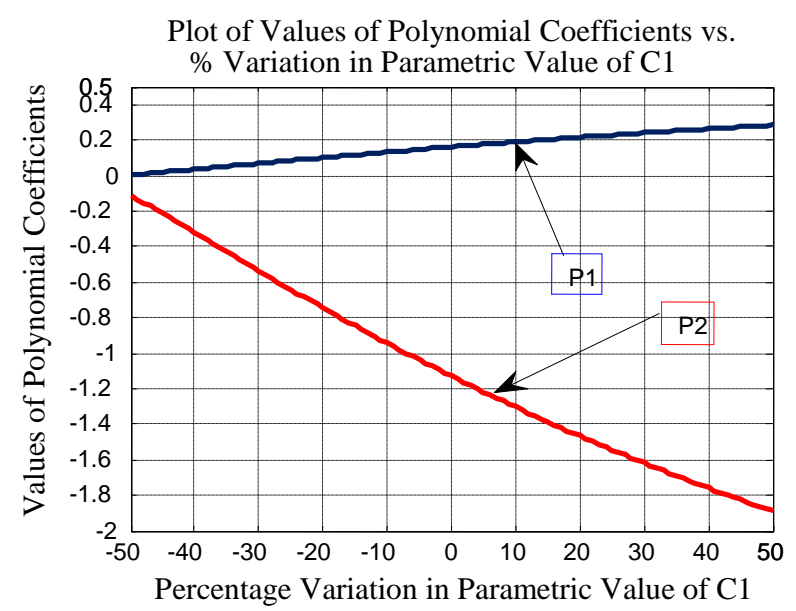

Plot of Values of Polynomial Coefficients vs. $\%$ Variation in Parametric Value of R1

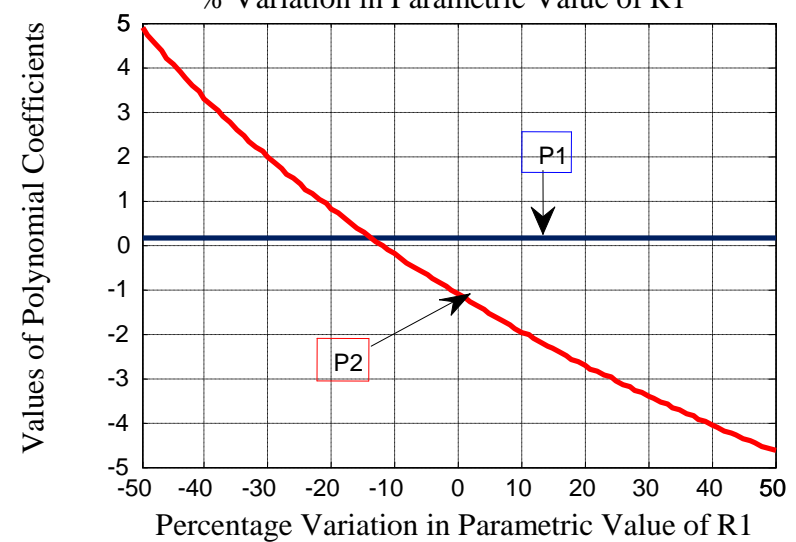

Plot of Values of Polynomial Coefficients vs. $\%$ Variation in Parametric Value of R3

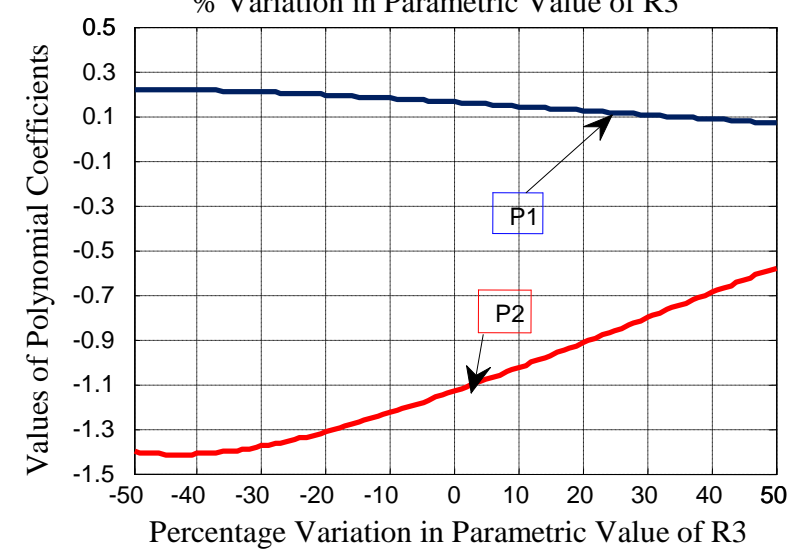

Plot of Values of Polynomial Coefficients vs. $\%$ Variation in Parametric Value of C2

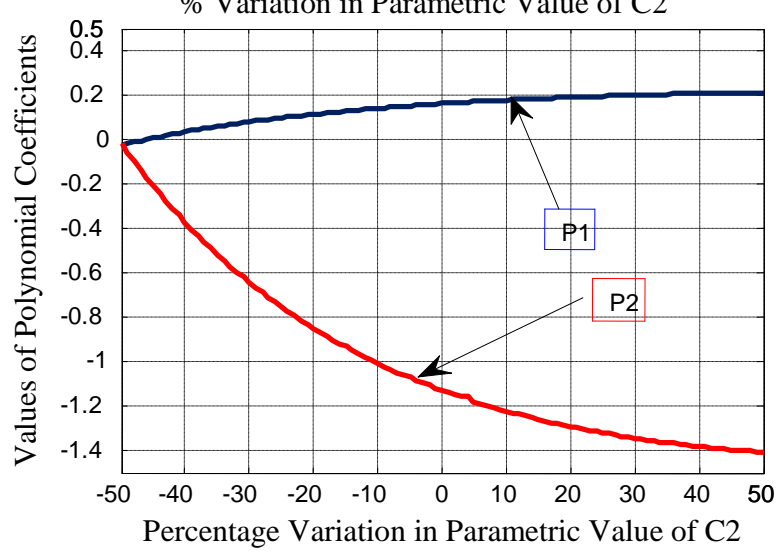

Plot of Values of Polynomial Coefficients vs. $\%$ Variation in Parametric Value of R2

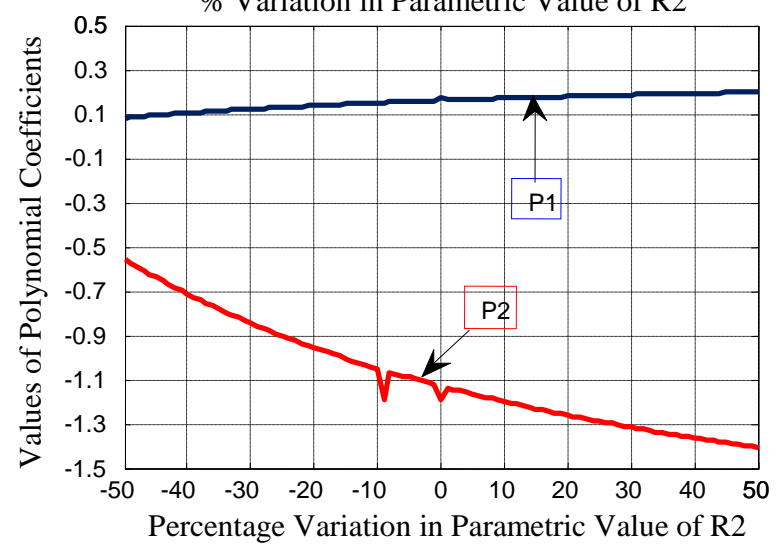

Plot of Values of Polynomial Coefficients vs. $\%$ Variation in Parametric Value of R4

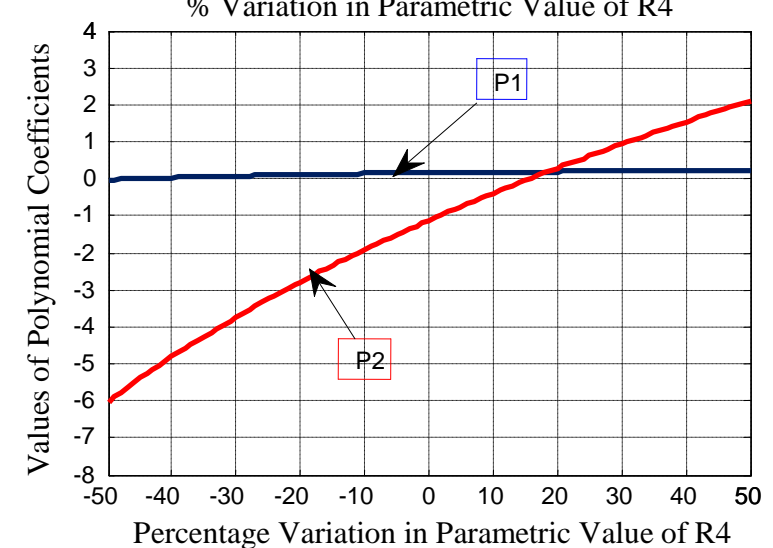

Fig 5: Plots of the fault dictionaries for the components fault models of circuit under test 


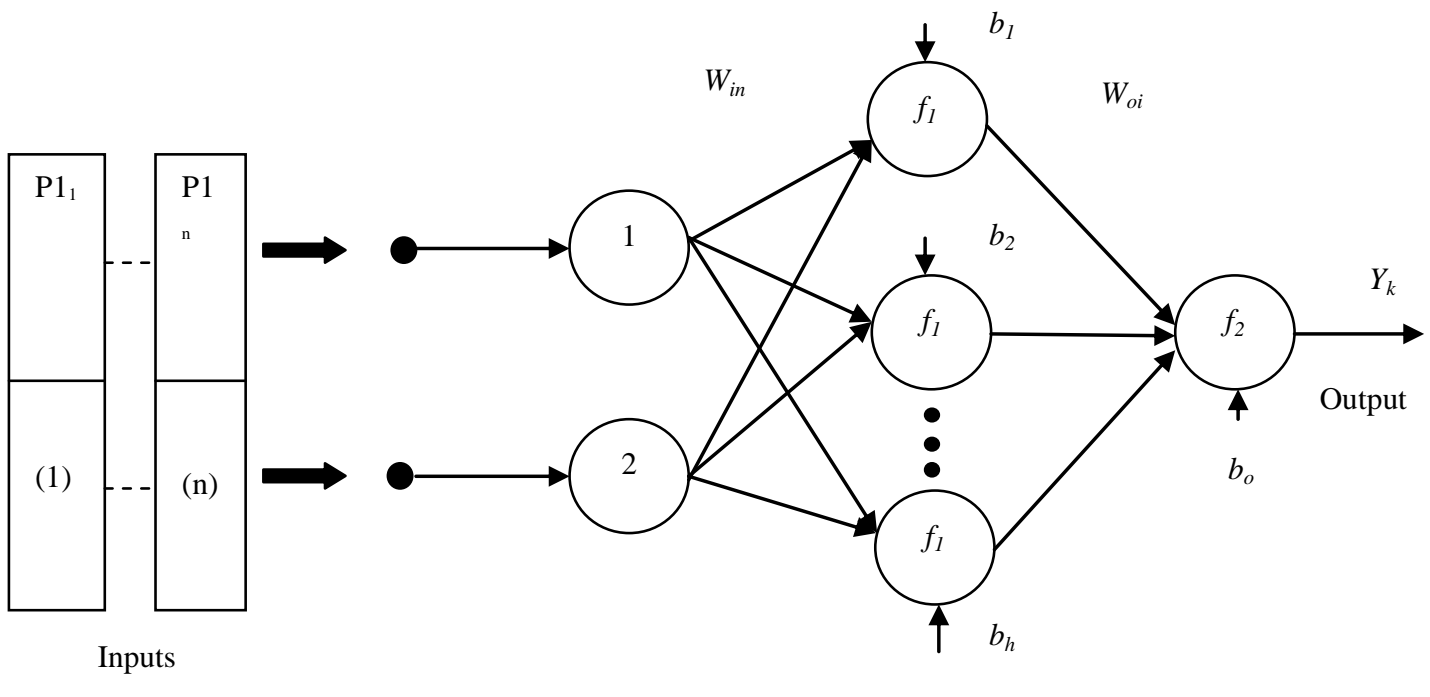

Fig 6: Proposed ANN model for the fault diagnosis of analog circuit.

The network is trained with Levenberg Marquardt training algorithm and the performance metric is taken as mean square error (MSE) and maximum absolute error. The mean square error is calculated as the difference between the target output and the network output. The goal is to minimize the average of the sum of these errors.

$M S E=\frac{1}{N} \sum_{k=1}^{N}\left(T_{k}-Y_{k}\right)^{2}$

Where $T_{k}$ is the target output and $Y_{k}$ is the neural network output.

The neural network with $10(X=10)$ nodes In the input layer and one node in the output layer is realized. Six neural network architecture each for one comonent under the diagnosis process is realized.The number of nodes in the hidden layer are varied to achieve the best fault classification performance. The total data collected in the dictionary is partitioned into three sets for training,validation and testing. Using the random selection for each of the component fault, 70 percent patterns are selected for training the neural network, 15 percent patterns are used for validation process and the remaining 15 persent patterns are used for testing purpose. Number of nodes in the hidden layer are varied till best classification evaluation is obtained. The best results of classification evaluation are obtained when the number of nodes in the hidden layer are as shown in table I. The network is adaptively trained to update the weights with LevenbergMarquardt backpropagation algorithm (trainlm) by the mean square error performance.

\section{RESULTS AND DISCUSSIONS}

The diagnosis results for the components $(\mathrm{C} 1, \mathrm{C} 2, \mathrm{R} 1, \mathrm{R} 2, \mathrm{R} 3$ and R4) of CUT are tabulated in table 1. The first metric used for fault classification is maximum absolute errror and the second is the mean square error for all three phases of the neural network classifier i.e. training, validation and testing phase.
Table 1: Fault diagnosis results for Bi Quad Filter Circuit

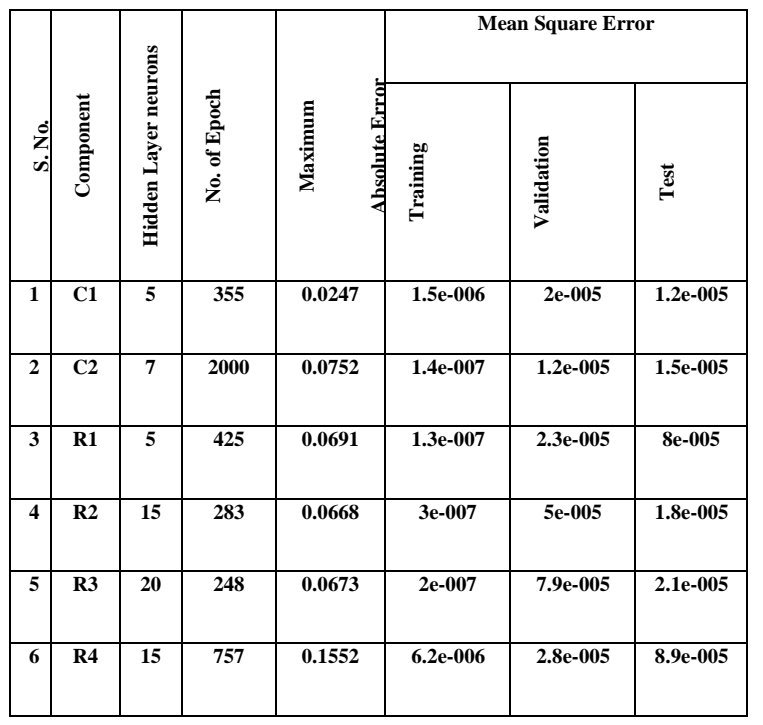

Maximum absolute error ranges from 0.0247 to 0.1552 . Mean squre error ranges from $1.3 \mathrm{e}-007$ to $6.2 \mathrm{e}-006,1.2 \mathrm{e}-005$ to $7.9 \mathrm{e}-005,1.5 \mathrm{e}-005$ to $8.9 \mathrm{e}-005$ for training, validation and test phases of neural network respectively. The neural network taraining, validation and test pattern performance plot for $\mathrm{C} 1$ is shown in figure7. The performance graph indicates that the best validation performance is obtained at 345 epochs.

In this section an analysis of the proposed methodology and its implementation for a practical example is also done. As it is clear from the results that one percent variation in the parametric value of the component is detectable with a maximum absolute error ranges from 0.0247 to 0.1552 . We have classified sucessfully all the faults of table I, except two R5 and R6, which are placed in ambugity group. This is because the output of the circuit overlap for these faults. 


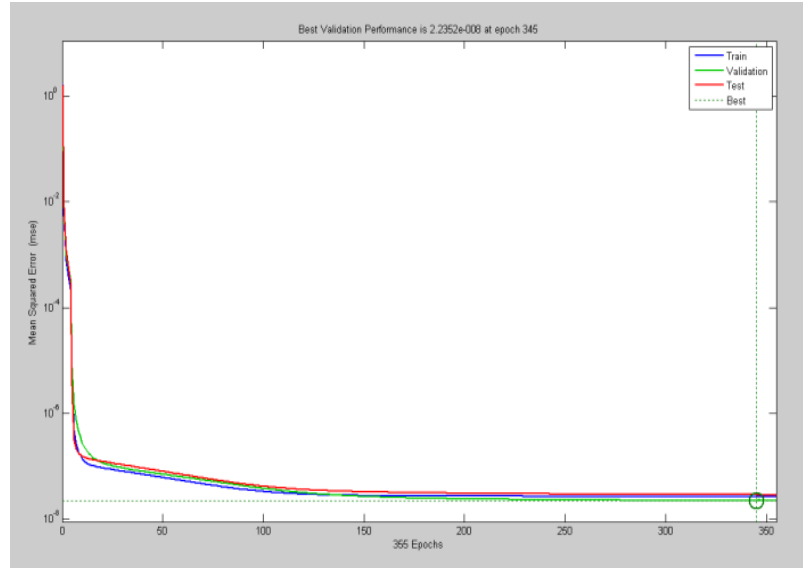

Fig 7: Performance plot of $\mathrm{NN}$ for capacitor $\mathrm{C} 1$

The results of the proposed methodology clearly indicate that through appropriate processing of analog circuit, one can train a neural network to correctly diagnose the single parametric faults with a fine variation in the parametric value of the component. This study indicates that the proposed preprocessing techniques have a significant imact on analog fault diagnosis, one due is ability to diagnose the single parametric faults of the components and secondly diagnosis with high sensitivity. The main contribution of this work is formulation and solving the fault diagnois problem in frequency domain. Hence in general, the method works correctly and gives results with minimum erorr as illustrated via example.

\section{CONCLUSION}

The use of the neural network, based upon the polynomial coefficients of the output frequency response of an analog circuit under test is presented in this paper. By performing the frequency response and curve fitting, polynomial coefficients are obtained for faulty and non faulty conditions of CUT. These polynomial coefficients are used as signature for the training, validation and test sets for artificial neural network. . The result of the proposed method applied to the Bi Quad Filter circuit is quite encouraging.

\section{ACKNOWLEDGEMENTS}

Authors are greatly indebted to the Department of Electronics and Communication Engineering, SLIET, Longowal-148106 (District: Sangrur), Punjab, India for providing excellent lab facilities that make this work feasible.

\section{REFERENCES}

[1] William G. Fenton, T. M. McGinnity, and Liam P.Maguire, Fault Diagnosis of Electronic Systems Using Intelligent Techniques: A Review, IEEE Transactions on Systems, Man, and Cybernetics-part c: Applications and Reviews 31, No.3 (2001) 269-281.

[2] Yuan Haiying, Chen Guangju and Xie Yongle, Feature Evaluation and Extraction Based on Neural Network in Analog Circuit Fault Diagnosis, Journal of Systems Engineering and Electronics 18, No. 2 (2007) 434-436.

[3] HU Mei, WANG Hong, Hu Geng and YANG Shiyuan, Soft Fault Diagnosis for Analog Circuits Based on Slope Fault Feature and BP Neural Networks, TSINGHUA SCIENCE AND TECHNOLOGY ISSN 1007-0214 05/49, 12, No. SI, (2007) 26-31.

[4] V. Manikandan and N. Devarajan, SBT Approach towards Analog Electronic Circuit Fault Diagnosis,
Hindawi Publishing Corporation, Active and Passive Electronic Components, ID 59856, (2007).

[5] M. A. El-Gamal and M. D. A. Mohamed, Ensembles of Neural Networks for Fault Diagnosis in Analog Circuits, Journal of Electronic Testing: Theory and Application 23 (2007) 323-339.

[6] Yin Shirong, Chen Guangju and Xie Yongle, Wavelet Neural Network Based Fault Diagnosis in Nonlinear Analog Circuits, Journal of System Engineering and Electronics 17 No. 3 (2006) 521-526.

[7] P. Kalpana , K. Gunavathi, Wavelet based fault detection in analog VLSI circuits using neural networks, ELSEVIER, Applied Soft Computing 8 (2008) 1592 1598.

[8] Mehran Aminian and Farzan Aminian, Neural-Network Based Analog-Circuit Fault Diagnosis Using Wavelet Transform as Preprocessor, IEEE Transactions on Circuits and Systems-II: Analog and Digital Signal Processing 47, No. 2 (2000) 151-156.

[9] Wenji Zhu, Yigang He, A Neural-Network-Based Fault Diagnosis Approach for Analog Circuits by Using Wavelet Transformation and Fractal Dimension as a Preprocessor, World Academy of Science, Engineering and Technology 68 (2010) 571-579

[10] A.Rathinam, R.Srinivasa Raghavan and R.Venkatraman, Fault Diagnosis in Analog Integrated Circuits Using Artificial Neural Networks, International Journal of Computer Applications (0975 - 8887) 1 No. 27 (2010) 63-69.

[11] M. F. Abu El-Yazeed and Adel A. K.Mohsen, A Preprocessor for Analog Circuit Fault Diagnosis Based on Prony's Method, AEU International Journal of Electronics and Communications (2003) 16-22.

[12] Marcantonio Catelani, Ada Fort, Cesare Alippi, A fuzzy approach for soft fault detection in analog circuits, ELESEVIER, Measurement 32 (2002) 73-83.

[13] K. Mohammadi, S.J. Seyyed Mahdavi , On improving training time of neural networks in mixed signal circuit fault diagnosis applications, ELSEVIER , Microelectronics Reliability 48 (2008) 781-793.

[14] Vanco Litovski, Miona Andrejevic, and Mark Zwolinski, Analogue Electronic Circuit Diagnosis Based on ANNs, Microelectronics Reliability 46 (2006) 1382-1391.

[15] Marcantonio Catelani and Ada Fort, Soft Fault Detection and Isolation in Analog Circuits: Some Results and a Comparison between a Fuzzy Approach and Radial Basis Function Networks, IEEE Transactions on Instrumentation and Measurement 51, No. 2 (2002) 196202.

[16] Doried Mismar,and Ayman AbuBaker, Neural Network Based Algorithm of Soft Fault Diagnosis in Analog Electronic Circuits, IJCSNS International Journal of Computer Science and Network Security,10 No.1 (2010) 107-111.

[17] Kaminska, B.; Arabi, K.; Goteti, P.; Huertas, J.; Kim, B.; Rueda, A.; and Soma, M., Analog and mixed signal benchmark circuits. First release, IEEE Proceedings International Test Conference (1997).

[18] Balivada, A.; Chen, J.; and Abraham, J., Analog testing with time response parameters. IEEE Design and Test of computers, (1996) 18-25. 